\title{
Saturation of the response to stochastic forcing in two-dimensional backward-facing step flow: A self-consistent approximation
}

\author{
Vladislav Mantič-Lugo* and François Gallaire ${ }^{\dagger}$ \\ Laboratory of Fluid Mechanics and Instabilities, École Polytechnique Fédérale de Lausanne, \\ EPFL-STI-IGM-LFMI, CH-1015, Switzerland \\ (Received 12 January 2016; published 21 December 2016)
}

\begin{abstract}
Selective noise amplifiers are characterized by large linear amplification to external perturbations in a particular frequency range despite their global linear stability. Applying a stochastic forcing with increasing amplitude, the response undergoes a strong nonlinear saturation when compared to the linear estimation. Building upon our previous work, we introduce a predictive model that describes this nonlinear dynamics, and we apply it to a canonical example of selective noise amplifiers: the backward-facing step flow. Rewriting conveniently the stochastic forcing and response in the frequency domain, the model consists in a mean flow equation coupled to the linear response to forcing at each frequency. This coupling is attained by the Reynolds stress, which is constructed by the integral in frequency of the independent responses. We generalize the model for a response to a white noise forcing $\delta$-correlated in space and time restricting the flow dynamics to its most energetic patterns calculated from the optimal harmonic forcing and response of the flow. The model estimates accurately the response saturation when compared to direct numerical simulations, and it correctly approximates the structure of the response and the mean flow modification. It also shows that the response undergoes a selective process governed by the nonlinear gain, which promotes a response structure with an approximately single frequency and wavelength in the whole domain. These results suggest that the mean flow modification by the Reynolds stress is the key nonlinearity in the saturation process of the response to white noise.
\end{abstract}

DOI: 10.1103/PhysRevFluids.1.083602

\section{INTRODUCTION}

A wide variety of open flows are characterized by their linearly stable nature while presenting high sensitivity to background disturbances. Typically, this behavior is encountered in boundary layers, mixing layers, jets, or separated flows even in the laminar regime at low and intermediate (preturbulent) Reynolds numbers. Such behavior is produced by convective instabilities and interpreted by the non-normality of the Navier-Stokes system of equations, which is able to amplify perturbations while being advected downstream by means of nonmodal mechanisms [1,2]. These flows are sometimes denoted as selective noise amplifiers due to their ability to amplify perturbations in particular frequency ranges.

A substantial body of work has been devoted to the study of amplifiers. Since classical modal analysis focuses on the eigenvalues and eigenmodes of the linear operator $\mathscr{L}$ describing the linearized dynamics of the flow around a steady solution, it fails to capture transient and forced behaviors in stable amplifier flows. Therefore, nonmodal techniques derived from classical linear algebra are used to characterize the physical behavior and amplification potential in linearly stable flows. One of the standard approaches to characterize the amplifier dynamics is to look at initial disturbances that lead to the maximum growth and follow the time evolution of these perturbations, described by the leading singular vectors of the time propagator $e^{\mathscr{L}_{t}}$ [3-5]. Studies of optimal initial perturbation have

\footnotetext{
*vladislav.manticlugo@epfl.ch

${ }^{\dagger}$ francois.gallaire@epfl.ch
} 
been carried out in parallel flows, e.g., [2,6,7], as well as in nonparallel flows, for example in spatially developing boundary layers [8-11] or in the backward-facing step flow [12]. Another alternative consists in finding the optimal harmonic forcing structures that at frequency $\omega$ lead to the most energetic responses. The optimal forcing and corresponding response are described by the singular vector of the resolvent operator $\mathscr{R}(\omega)=(i \omega+\mathscr{L})^{-1}[2,4]$. Optimal forcing/response structures have been assessed in plane Couette [13] as well as in spatially developing open flows [8,10,11,14] and particularly in the backward-facing step [15-19]. A slightly different approach is undertaken by Garnaud, Lesshaftt, Schmid, and Huerre [14] where, in an attempt to describe more precisely the actual physics involved in the strong noise amplification exhibited in turbulent jets, they apply the optimal gain analysis on a model mean flow instead of the stable steady solution of the Navier-Stokes equations (NSEs) as in the previously mentioned studies. In general, both time and frequency approaches describe the most energetic instability mechanisms at play.

Realistic flows are in general subject to unpredictable noise created from different possible sources such as residual turbulence, acoustic disturbances, geometrical defects, etc. In this context, Farrell and Ioannou [20] have studied the response to white noise forcing in parallel flows, reformulating the linear problem as a Lyapunov equation for the covariance matrix that describes the statistically steady state of the response. Following this approach, they later obtained a low order approximation of the linear dynamical system for a Couette flow forced by white noise by extracting the energy ranked coherent structures of the stochastic response and forcing, the so-called empirical orthogonal functions (EOFs) and stochastic optima (SOs), respectively [21]. Dergham, Sipp, and Robinet [17] introduced a low dimensional model to describe the linear behavior of the flow around the backwardfacing step forced by white noise. The low dimensional model is constructed by the mentioned EOFs and SOs, extracted from the most energetic harmonic forcing/response structures, relating the stochastic structures to the harmonic optima. Additionally, the response to inlet white noise forcing in the backward-facing step shows that the exact stochastic response from the direct numerical simulation (DNS) is well characterized by the two-dimensional (2D) optimal perturbances [12]. More recently, Boujo and Gallaire [18] have studied the sensitivity of the stochastic response to passive forcing devices, with control applications in mind.

These studies are limited to the linear characterization of the flow behavior, thus failing to describe saturation processes or nonlinear interactions involved in the transition to turbulence in linearly stable flows. In many cases, the non-normality of the NSE allows the flow to escape from linearly stable solutions by means of large amplification of external disturbances. Several models with a coupling between the mean flow and the linear perturbation equation have been proposed. The stochastic structural stability theory (SSST) has been introduced by Farrell and Ioannou [22,23], consisting in a coupled system of equations where the linear response to white noise forcing rewritten as a Lyapunov equation [20] is coupled to the slowly varying ensemble average mean flow by means of the Reynolds stress. This theory is able to describe sustained coherent structures that appear during the transition to turbulence in the 3D Couette flow [23] as well as in turbulent atmospheric flows [22,24]. As an alternative, the reduced nonlinear model (RNL) approach has been proposed [25] coupling the mean flow to a single realization of the stochastic linear response, avoiding the computation of the Lyapunov equation [23]. Another semilinear approach was recently proposed to describe the coherent structures appearing in the transition to turbulence for parallel shear flow [26]. Most of these nonlinear models have been devoted to the study of coherent sustained structures in turbulent flows, but a formalized quantitative physical description of the dynamics involved in the saturation of strong amplifiers under stochastic excitations is still missing.

Motivated by the SSST [22,23] and the low order modeling based on harmonic optima [17], we propose a model to describe the nonlinear dynamics of the response to white noise forcing in a strongly amplifying flow, with the objective to capture its saturation with an increasing forcing amplitude. The model is applied to a canonical amplifier flow, the incompressible backward-facing step flow, which is archetypical in fundamental studies of flow separation induced by abrupt changes of geometry. The flow is globally stable at the Reynolds numbers considered, $\operatorname{Re}=500$ and 700 [27,28]. The work presented herein is an extension to white noise forcing of the self-consistent 


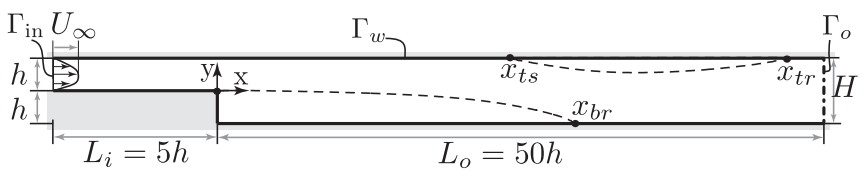

FIG. 1. Sketch of the flow configuration of the backward-facing step with two recirculation bubbles at $\operatorname{Re}=500$, one at the top and one at the bottom.

model [19] previously introduced for the nonlinear saturation of the response to harmonic forcing. In that model, the mean flow is coupled to a linear response to harmonic forcing around the mean flow by means of the Reynolds stress, neglecting the nonlinear interaction of the response with itself [19]. In the present study, we reformulate the self-consistent model in the frequency domain to account for the stochastic nonlinear response to a band-limited $\delta$-correlated white noise. The results aim at clarifying whether the nonlinear stochastic response can be well approximated linearly in the frequency domain and the role of the Reynolds stress and the mean flow distortion in the saturation mechanism, as discussed in the literature [29-33].

The paper is structured as follows: Section II introduces the flow configuration and the linear description of the response. Section III describes the temporal stochastic forcing, introduces the model, and provides a comparison with DNS results. The model is adapted to stochastic spatiotemporal forcing in Sec. IV, before conclusions are drawn in Sec. V.

\section{PROBLEM FORMULATION}

\section{A. Flow configuration and governing equations}

We consider the response to forcing of a laminar incompressible flow around the 2D backwardfacing step. The flow configuration is depicted in Fig. 1. An inlet channel with height $h$ and length $L_{i}=5 h$ precedes a step of height $h$ and expands into a wider channel of height $H=2 h$ and length $L_{o}$, hence determining an expansion ratio $\Xi=h / H=0.5$. The inlet boundary condition at $\Gamma_{\text {in }} \in[0 ;+1]$ is a plane Poiseuille profile $\boldsymbol{u}_{\text {Pois }}$ with a centerline (maximum) velocity $U_{\infty}$ plus a general inlet forcing $f$. The centerline velocity $U_{\infty}$ defines the Reynolds number $\operatorname{Re}=U_{\infty} h / v$, where $h$ is the inlet height and $v$ is the kinematic viscosity. A Cartesian coordinate system is adopted with $x$ in the streamwise direction and $y$ in the wall-normal direction, with unitary vectors $\boldsymbol{e}_{x}$ and $\boldsymbol{e}_{y}$. A no-slip boundary condition is imposed on the side walls $\Gamma_{w}$ and an outflow condition at the end of the domain $\Gamma_{o} \in[-1 ;+1]$. These boundaries are omitted for brevity in the sequel. The nondimensional frequency is defined by the Strouhal number $\mathrm{St}=f_{H z} h / U_{\infty}$ and related to the nondimensional angular frequency as $\mathrm{St}=\omega / 2 \pi$.

The flow is governed by the 2D incompressible Navier-Stokes equations (NSEs),

$$
\begin{aligned}
\nabla \cdot \boldsymbol{u} & =\mathbf{0}, \\
\partial_{t} \boldsymbol{u}+\mathscr{N}(\boldsymbol{u}) & =0, \\
\boldsymbol{u} & =\boldsymbol{u}_{\mathrm{Pois}}+\boldsymbol{f} \text { on } \Gamma_{\mathrm{in}},
\end{aligned}
$$

where

$$
\mathscr{N}(\boldsymbol{u}) \equiv(\boldsymbol{u} \cdot \nabla) \boldsymbol{u}+\nabla p-\mathrm{Re}^{-1} \Delta \boldsymbol{u}
$$

collects the advective, pressure gradient, and diffusive contributions. The pressure field $p$ is such that the velocity field is divergence-free, $\boldsymbol{\nabla} \cdot \boldsymbol{u}=\mathbf{0}$, following the incompressibility condition.

The Navier-Stokes equations are solved using the finite element method with the flow fields $\left(u_{x}, u_{y}, p\right)$ spatially discretized by Taylor-Hood $(\mathrm{P} 2, \mathrm{P} 2, \mathrm{P} 1)$ elements. The software FREEFEM++ [34] is used to generate the domain $\Omega$ triangulation and to build all the required operators. The steady solutions of the nonlinear systems of NSEs are computed using the Newton-Raphson method, while 
the time varying DNSs of the NSEs are integrated using a second order characteristics-Galerkin method. Further details on the numerical approach can be found in Ref. [19].

\section{B. Linear transfer function}

The steady solution of NSEs defines the base flow $\boldsymbol{U}_{B}$,

$$
\mathscr{N}\left(\boldsymbol{U}_{B}\right)=0,
$$

characterized by two recirculation bubbles illustrated in Fig. 1 for the backward-facing step at the chosen $\operatorname{Re}=500$, as studied in the literature $[12,15,18,27]$. For an expansion ratio $\Xi=0.5$, the flow is globally stable at this Reynolds number, presenting mainly a 2D response to white noise [12] and thus supporting the choice of a 2D analysis. The threshold for the 3D global instability was found to be $\operatorname{Re}_{\mathrm{cr}} \sim 748[27,28]$.

Assuming a small amplitude of the forcing, the exact nonlinear response can be approximated linearly as

$$
\begin{aligned}
{\left[\partial_{t} \boldsymbol{u}_{1 B}^{\prime}+\mathscr{L}_{\boldsymbol{U}_{B}}\left(\boldsymbol{u}_{1 B}^{\prime}\right)\right] } & =0, \\
\boldsymbol{u}_{1 B}^{\prime} & =\boldsymbol{f} \text { on } \Gamma_{\mathrm{in}},
\end{aligned}
$$

where higher order nonlinear terms are neglected, and the operator $\mathscr{L}_{\boldsymbol{U}}\left(\boldsymbol{u}^{\prime}\right)$ is the corresponding operator for the NSE linearized around any $\boldsymbol{U}$, i.e.,

$$
\mathscr{L}_{\boldsymbol{U}}\left(\boldsymbol{u}^{\prime}\right) \equiv(\boldsymbol{U} \cdot \nabla) \boldsymbol{u}^{\prime}+\left(\boldsymbol{u}^{\prime} \cdot \nabla\right) \boldsymbol{U}+\nabla p^{\prime}-\operatorname{Re}^{-1} \Delta \boldsymbol{u}^{\prime}
$$

While the focus of the study is on the response to stochastic forcing, we describe first the harmonic response to facilitate understanding and to predict in which frequency range larger amplifications are more likely to be observed [4,5,17-19]. For a harmonic forcing $\boldsymbol{f}(y, t)=\boldsymbol{f}_{1}(y) e^{i \omega t}+$ c.c. with a spatial distribution $\boldsymbol{f}_{1}(y)$ and frequency $\mathrm{St}=\omega /(2 \pi)$, the corresponding linear response $\boldsymbol{u}^{\prime}(x, t)$ is also harmonic, $\boldsymbol{u}^{\prime}(x, t) \simeq \boldsymbol{u}_{1 B}^{\prime}(x, t)=\boldsymbol{u}_{1 B}(x) e^{i \omega t}+$ c.c., and it oscillates at the forcing frequency, due to the linearity of the operator (4). The linear equation (4) can be rewritten formally as $\boldsymbol{u}_{1 B}=\mathscr{R}_{B}(\omega) \boldsymbol{f}_{1}$, where $\mathscr{R}(\omega)=\left(i \omega \mathscr{I}+\mathscr{L}_{\boldsymbol{U}}\right)^{-1}$ is the resolvent operator for any steady $\boldsymbol{U}$ and $\mathscr{R}_{B}(\omega)=\left(i \omega \mathscr{I}+\mathscr{L}_{\boldsymbol{U}_{B}}\right)^{-1}$ is the resolvent operator for the base flow.

A natural way of measuring the amplification of the nonlinear dynamical system is the gain, which is defined as the ratio between the amplitude of the output response and the amplitude of the input forcing. It is equal to the square root of the ratio of the energy of the output response to the energy of the input forcing. For the specific linear case of harmonic forcing, it reads

$$
G_{B}(\omega)=\frac{\left\|\boldsymbol{u}_{1 B}\right\|_{\Omega}}{\left\|\boldsymbol{f}_{1}\right\|_{\Gamma_{\text {in }}}}=\frac{\left\|\mathscr{R}_{B}(\omega) \boldsymbol{f}_{1}\right\|_{\Omega}}{\left\|\boldsymbol{f}_{1}\right\|_{\Gamma_{\text {in }}}},
$$

where the $L^{2}$ norm $\|$.$\| is determined by the Hermitian inner product (\boldsymbol{a} \mid \boldsymbol{b})=\int_{\Omega} \overline{\boldsymbol{a}} \cdot \boldsymbol{b} d \Omega$ for complex fields in the domain $\Omega$, with straightforward restriction on the boundary $\Gamma_{\text {in }}$.

The linear gain $G_{B}(\omega)$ around the base flow is a function of the forcing frequency $\omega$, as illustrated in Fig. 2(a), for the response to a forcing in the form of a Poiseuille profile $\boldsymbol{f}_{1}=y(1-y)$. The gain $G_{B}(\omega)$ follows a bell-shaped curve with a maximum at $\mathrm{St}_{\mathrm{opt}}=\omega_{\mathrm{opt}} / 2 \pi=0.075$. Furthermore, both the amplitude of the response and the shape of the response depend on the forcing frequency $\omega$, as shown in the velocity contours presented in Fig. 2(b). The structure of the response displays an apparent characteristic wave number that increases with the frequency, and an envelope that migrates downstream for larger gains $[16,18]$.

The linear response to white noise forcing would correspond to a combination of all the different response structures multiplied by their corresponding amplitudes since a pure white noise forces all the frequencies with the same energy. 
(a)

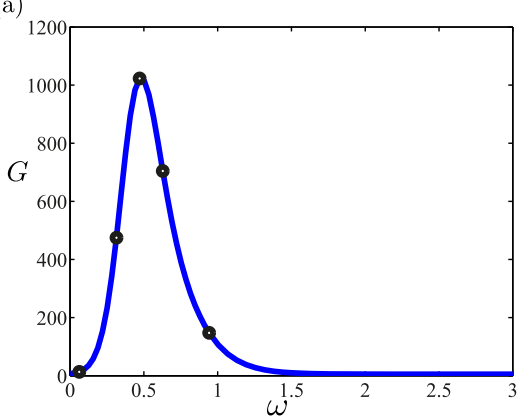

(b)

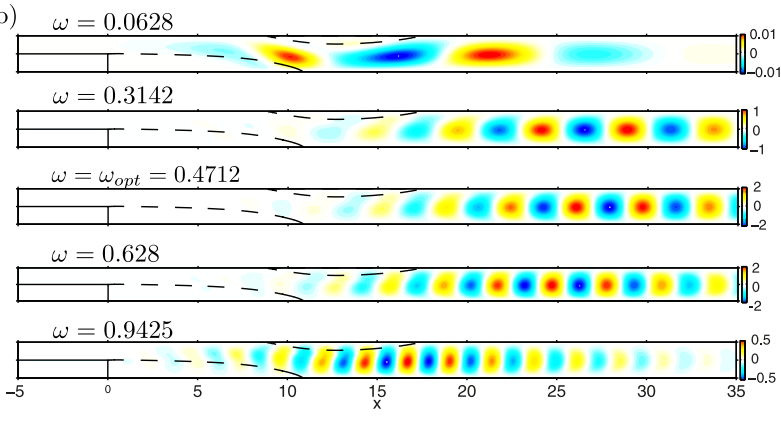

FIG. 2. (a) Linear gain $G_{B}(\omega)$ around the base flow and (b) contours of the velocity in the $y$-direction of the linear response for different forcing frequency $\mathrm{St}=\omega / 2 \pi$ for a harmonic forcing of the form of a Poiseuille profile $\boldsymbol{f}_{1}=y(1-y)$. The linear gain of the selected frequencies in (b) is marked as black circles in the curve in (a). The maximal gain is attained at the optimal frequency $\mathrm{St}=\omega_{\mathrm{opt}} /(2 \pi)=0.075$. Plots for the backward-facing step at $\operatorname{Re}=500$.

\section{TEMPORAL STOCHASTIC FORCING}

\section{A. Forcing definition and white noise response}

In more realistic cases, the external disturbances are more likely characterized by a broadband frequency rather than being harmonic. Thereby, to model these physical perturbations, we excite the flow by a random noise, characterized by its statistical properties. Nonetheless, there are advantages in addressing the study of the response to stochastic forcing in the frequency domain $[4,17]$. The power spectral density function (PSD) characterizes the energy distribution of the input signal in the frequency domain. With the aid of a truncated Fourier transform for a time signal $x(t)$ of length $[0, T]$,

$$
\hat{x}_{T}(\omega)=\frac{1}{\sqrt{T}} \int_{0}^{T} x(t) e^{-i \omega t} d t
$$

the PSD is defined as

$$
S_{x x}(\omega)=\left|\hat{x}_{T}(\omega)\right|,
$$

which, in the limit $T \rightarrow \infty$, converges to the expected value of $\hat{x}(\omega), \lim _{T \rightarrow \infty} S_{x x}(\omega)=\mathscr{E}\left[|\hat{x}(\omega)|^{2}\right]$. In general, a white noise signal $\xi(t)$ is $\delta$-correlated, $\left\langle\xi(t) \xi^{\dagger}(s)\right\rangle=\delta(t-s)$, and defined by a constant $\operatorname{PSD} S_{\xi \xi}(\omega)=|\hat{\xi}|^{2}=S$ with infinite power $P$. Indeed, the power is defined as

$$
P=\lim _{T \rightarrow \infty} \frac{1}{T} \int_{0}^{T}\left|\xi_{T}(t)\right|^{2} d t=\frac{1}{\pi} \int_{0}^{\infty}|\hat{\xi}|^{2} d \omega=\sigma^{2}
$$

thanks to Parseval's theorem and to the definition of the variance $\sigma$. Because $S>0$, a pure white noise has infinite power and is not physically realizable being an idealization of physical noises. Physical systems usually are band-limited and are affected by the noise within this band.

A digital random signal $\xi_{d}(t)$ has a natural band-limiting frequency given by its sampling time step, $\omega_{d} /(2 \pi)=1 / 2 \delta t$. To obtain time step independent results, the signal is filtered to a bandlimiting frequency $\omega_{b}$ yielding a power and variance $P_{b}=\sigma_{b}^{2}=\left|\hat{\xi}_{b}\right|^{2} \omega_{b} / \pi$. Figure 3(a) compares a realization of the unfiltered sampling-limited white noise signal with unit variance and power and the filtered noise with a band-limiting frequency $\omega_{b} / 2 \pi=1$. Figure $3(\mathrm{~b})$ compares the PSD for the actual signals and their theoretical values. The PSD is estimated using a Welch method in MATLAB.

The inlet forcing used in the study is defined as $\boldsymbol{f}=A \boldsymbol{f}_{1}(y) \xi_{b}(t)$, stochastic in time by the function $\xi_{b}(t)$, a band-limited white noise, $\delta$-correlated with zero mean, and unit power and variance $\sigma=1$, with constant PSD $2\left|\hat{\xi}_{b}\right|^{2}=2 \pi / \omega_{b}$ that depends only on the band-limiting frequency. For 

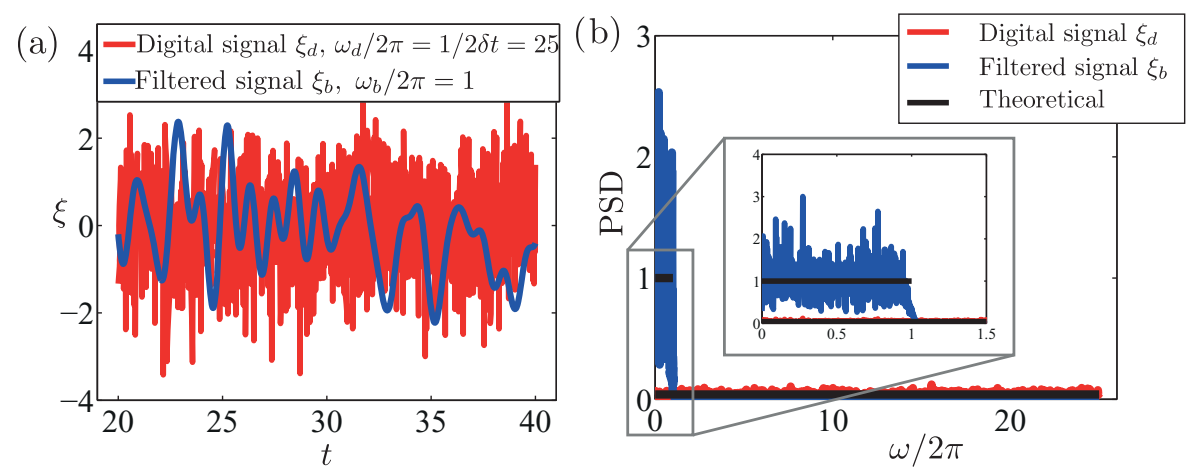

FIG. 3. (a) Realization of a white noise signal with unit power $P=1$, comparing a signal without filtering $\omega_{b} / 2 \pi=1 /(2 \delta t)=25$ and a filtered one with a band-limiting frequency $\omega_{b} / 2 \pi=1$. (b) Comparison of the power spectral density for these two signals.

the sake of simplicity, we start with a fixed spatial distribution in the form of a Poiseuille profile $\boldsymbol{f}_{1}(y)=\sqrt{30} y(1-y)$, such that $\int_{\Gamma_{\text {in }}} \boldsymbol{f}_{1}(y)^{2} d \Gamma_{\text {in }}=1$ and $A$ is the amplitude of the forcing. The choice of the forcing structure is based on simplicity since the methodology presented in the paper is general and independent of the forcing shape, affecting only the value of the gain and minimally the response structure. Defining $\langle\cdot\rangle=\frac{1}{T} \int_{0}^{T} d t$ as the time average over a time span $T$ long enough to achieve $T$ independent results, the power of the forcing relates to its amplitude as

$$
\left\langle\int_{\Gamma_{\text {in }}} \boldsymbol{f}^{2} d \Gamma_{\text {in }}\right\rangle=\frac{1}{\pi} \int_{0}^{\omega_{b}}\left\|\boldsymbol{f}_{1}\right\|_{\Gamma_{\text {in }}}^{2}\left|\hat{\xi}_{b}\right|^{2} A^{2} d \omega=A^{2} .
$$

The amplitude of the response or variance is defined in general through

$$
R^{2}=\left\langle\int_{\Omega} \boldsymbol{u}^{\prime 2} d \Omega\right\rangle=\frac{1}{\pi} \int_{0}^{\omega_{b}} G^{2}(\omega)\|\hat{\boldsymbol{f}}\|_{\Gamma_{\mathrm{in}}}^{2} d \omega=\frac{A^{2}}{\omega_{b}} \int_{0}^{\omega_{b}} G^{2}(\omega) d \omega,
$$

where $\boldsymbol{u}^{\prime}=\boldsymbol{u}-\langle\boldsymbol{u}\rangle$ is the pure fluctuating velocity with zero mean $\left\langle\boldsymbol{u}^{\prime}\right\rangle=0, G(\omega)$ is the gain at each frequency, and $R$ is the amplitude of the response.

For the sake of clarity, we describe a complementary point of view, which consists in fixing the PSD of the white noise forcing $2\left|\hat{\xi}_{b}\right|^{2}=S=\mathrm{cst}$, and thus allowing the power $P\left(\omega_{b}\right)$ to vary with the band-limiting frequency $\omega_{b}$. In this setting, the amplitude of the linear response $R$ tends asymptotically to a constant value for the infinite limit of the band frequency $\omega_{b} \rightarrow \infty$ and thus requires infinite power $P \rightarrow \infty$ of the forcing, as shown in Fig. 4(a). This asymptotic behavior to a constant limit follows from the gain curve $G(\omega)$, which tends to zero, $\lim _{\omega \rightarrow \infty} G(\omega)=0$ [4], as illustrated in Fig. 2, where large amplifications are only concentrated at low frequencies $\omega$. It should be highlighted that this behavior persists also in nonlinear systems, which can be described by a nonlinear gain, since in general, physical systems damp high frequencies. As classically used in the literature [4,17,20,23], the asymptotic response to pure white noise forcing $\omega_{b} \rightarrow \infty$ can therefore be accurately approximated by means of a more physical forcing with a band-limited white noise, provided that the band-limiting frequency $\omega_{b}$ is far enough from the low frequency amplification region (Fig. 2). This holds true for $\omega_{b} /(2 \pi)=1$, which is used in all of the sequel.

The total nonlinear gain for the stochastically forced system reads as the ratio between the amplitude of the response and the amplitude of the forcing. It is related to the ratio of the power of the fluctuating response $\boldsymbol{u}^{\prime}$ to the power of the forcing $\boldsymbol{f}$, as defined in Eqs. (11) and (10)

$$
G_{\mathrm{tot}}^{2}=\frac{\left\langle\int_{\Omega} \boldsymbol{u}^{\prime 2} d \Omega\right\rangle}{\left\langle\int_{\Gamma_{\mathrm{in}}} \boldsymbol{f}^{2} d \Gamma_{\mathrm{in}}\right\rangle}=\frac{1}{\omega_{b}} \int_{0}^{\omega_{b}} G^{2}(\omega) d \omega .
$$



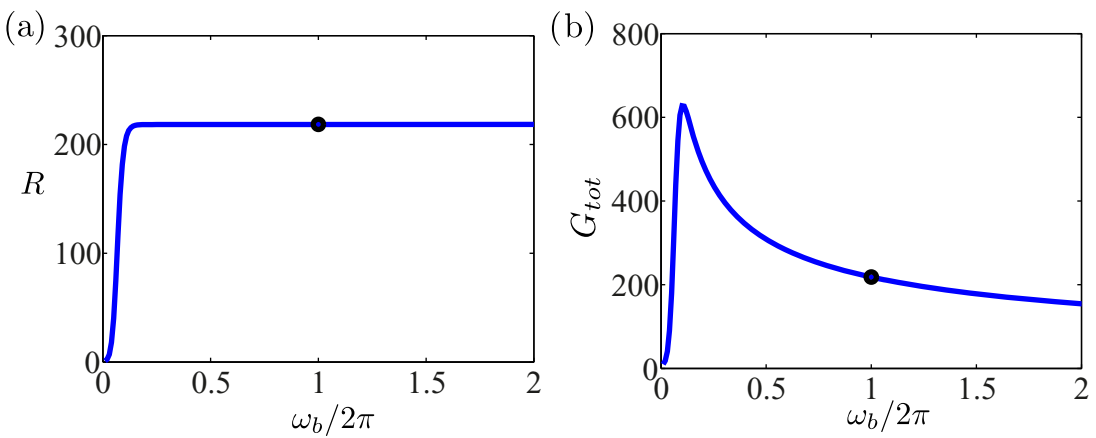

FIG. 4. (a) Amplitude of the linear response $R$ and (b) total linear gain $G_{\text {tot }}$ as a function of the band-limiting frequency $\omega_{b}$ for a band-limited white noise with constant PSD $2\left|\hat{\xi}_{b}(\omega)\right|^{2}=1$ and varying power $P\left(\omega_{b}\right)(9)$, where the spatial distribution is in the form of a Poiseuille profile $f_{1}=\sqrt{30} y(1-y)$. The response amplitude and total gain are computed from the integration of the linear gain $G_{B}(\omega)$ around the base flow for the backward-facing step at $\operatorname{Re}=500$.

The total gain tends to zero, $\lim _{\omega_{b} \rightarrow \infty} G_{\text {tot }}=0$, for the limiting case of white noise as illustrated in Fig. 4(b), since an increase of the band-limiting frequency $\omega_{b}$ entails an increase in the power spent at higher frequencies, which have such a small amplification $G(\omega)$ (see Fig. 2) that they do not contribute to the power of the response $R^{2}$, while spending power in the forcing $A^{2}$. Actually, $G(\omega)$ should decrease faster than $\omega^{-1 / 2}$ in the large $\omega$ limit for the integral (12) to converge.

The backward-facing step presents a strong linear amplification of the forcing due to the nonnormality of the linear operator $\mathscr{L}_{\boldsymbol{U}_{B}}[15,18,19]$, as can be seen in Fig. 2 . This strong amplification limits the validity of the linear response to very small amplitude of the forcing. Therefore, one expects saturation to occur, which restrains the amplitude of the response for an increase of the forcing amplitude. This nonlinear saturation of the flow under stochastic forcing calculated by DNS can be appreciated in Fig. 5, where the total nonlinear gain $G_{\text {tot }}$ strongly reduces as the amplitude of the forcing $A$ increases. Along with the amplitude saturation, the response exhibits a change in structure with a migration upstream related to an increase in the forcing amplitude. This migration is connected to a shortening of the mean recirculation bubble, which is reminiscent the mean flow correction in the cylinder flow caused by the limit-cycle amplitude saturation $[32,33,35]$.
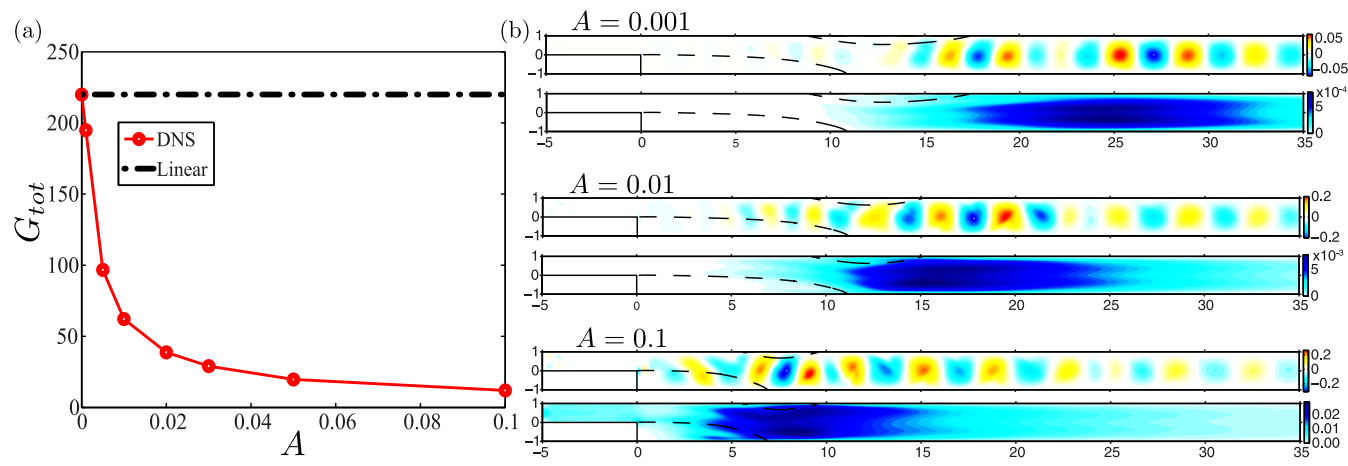

FIG. 5. Nonlinear total gain from DNS $G_{\text {tot }}$ and linear total gain $G_{B \text { tot }}$ as a function of the amplitude of the forcing $A$. The figure shows the saturation of the gain and the variation of the response structure. The insets show the perturbation velocity in the $y$-direction and the perturbation energy. $\operatorname{Re}=500$. 


\section{VLADISLAV MANTIČ-LUGO AND FRANÇOIS GALLAIRE}

\section{B. Self-consistent model for a temporal stochastic forcing}

Saturation problems of a similar nature to that illustrated in Fig. 5 have been modeled by means of a coupled system of mean flow and linear fluctuation equations for the case of the response to harmonic forcing [19], and for the flow past a cylinder above linear instability onset [32,33]. Another related approach is undertaken in the SSST [23] where a time-varying ensemble averaged mean flow, rather than a time average mean flow, is coupled to a linear response to white noise. Following these studies, we introduce the Reynolds decomposition

$$
\boldsymbol{u}(\boldsymbol{x}, t)=\boldsymbol{u}^{\prime}(\boldsymbol{x}, t)+\boldsymbol{U}(\boldsymbol{x})=\boldsymbol{u}^{\prime}(\boldsymbol{x}, t)+\boldsymbol{U}_{B}(\boldsymbol{x})+\Delta \boldsymbol{U}(\boldsymbol{x}) .
$$

The instantaneous flow is expressed as a mean flow $\boldsymbol{U}=\langle\boldsymbol{u}\rangle$ plus a pure fluctuation $\boldsymbol{u}^{\prime}$ with zero mean $\left\langle\boldsymbol{u}^{\prime}\right\rangle=0$. The mean-flow correction is denoted by $\Delta \boldsymbol{U}$. Inserting the Reynolds decomposition in the full NSE, we obtain a set of two coupled equations,

$$
\begin{aligned}
\mathscr{N}(\boldsymbol{U}) & =-\left\langle\left(\boldsymbol{u}^{\prime} \cdot \nabla\right) \boldsymbol{u}^{\prime}\right\rangle, \\
\boldsymbol{U} & =\boldsymbol{u}_{\mathrm{Pois}} \text { on } \Gamma_{\mathrm{in}}, \\
\partial_{t} \boldsymbol{u}^{\prime}+\mathscr{L}_{\boldsymbol{U}}\left(\boldsymbol{u}^{\prime}\right) & =-\left(\boldsymbol{u}^{\prime} \cdot \nabla\right) \boldsymbol{u}^{\prime}+\left\langle\left(\boldsymbol{u}^{\prime} \cdot \nabla\right) \boldsymbol{u}^{\prime}\right\rangle, \\
\boldsymbol{u}^{\prime} & =\boldsymbol{f} \text { on } \Gamma_{\mathrm{in}},
\end{aligned}
$$

where the mean flow $\boldsymbol{U}$ arises as a result of the Reynolds stress forcing in the steady mean flow equation (14a), while the forced response equation (14b) governs the time-dependent fluctuating field $\boldsymbol{u}^{\prime}$. Note that no simplification has been carried out so far.

In semilinear models like SSST [23], RNL [25], and others [26], the fluctuation equation is approximated linearly, thus eliminating the right-hand side of (14b) while keeping the Reynolds stress nonlinearity [right-hand side of (14a)]. In the same spirit, our recent studies [19,32,33] seem to indicate that the nonlinear interaction of the fluctuation with itself gathered in the term $-\left(\boldsymbol{u}^{\prime} \cdot \nabla\right) \boldsymbol{u}^{\prime}+\left\langle\left(\boldsymbol{u}^{\prime} \cdot \nabla\right) \boldsymbol{u}^{\prime}\right\rangle$ has a negligible influence in the saturation process for certain flows. Therefore, this nonlinear interaction is also neglected in the present model while keeping the nonlinearity gathered in the Reynolds stress. This is the main hypothesis inherent to the model assuming that restricting the nonlinear dynamics to the Reynolds stress is sufficient to capture the flow behavior.

The time varying fluctuation $\boldsymbol{u}^{\prime}$ of the coupled system (14) is rewritten in the frequency domain using (7) and (9) for a band-limiting frequency $\omega_{b}$. Thereby, the total Reynolds stress forcing [right-hand side of (14a)] is rewritten as the frequency integral of the independent Reynolds stress forcings $2 \operatorname{Re}[(\overline{\hat{\boldsymbol{u}}} \cdot \nabla) \hat{\boldsymbol{u}}]$ constructed by the response at each frequency $\omega$. The cross terms between different frequencies disappear in the Reynolds stress forcing thanks to the orthogonality of the frequency basis. The self-consistent system is obtained by neglecting the nonlinear interaction of the fluctuation with itself [i.e., the right-hand side in Eq. (14b)],

$$
\begin{aligned}
\mathscr{N}(\boldsymbol{U}) & =-\frac{1}{\pi} \int_{0}^{\omega_{b}} 2 \operatorname{Re}[(\overline{\hat{\boldsymbol{u}}} \cdot \nabla) \hat{\boldsymbol{u}}] d \omega, \\
\boldsymbol{U} & =\boldsymbol{u}_{\text {Pois }} \text { on } \Gamma_{\mathrm{in}}, \\
i \omega \hat{\boldsymbol{u}}+\mathscr{L}_{\boldsymbol{U}}(\hat{\boldsymbol{u}}) & =0, \\
\hat{\boldsymbol{u}} & =\hat{\boldsymbol{f}} \text { on } \Gamma_{\text {in }} .
\end{aligned}
$$

The model is composed of a set of independent linear equations (15b) that describe the response to noise at all frequencies $\omega \in\left[0 ; \omega_{b}\right]$ coupled to the mean flow equation (15a) by means of the Reynolds stress forcing. 
The integral of the Reynolds stress forcing in the frequency domain is then approximated by a discrete integral in a given set of discrete frequencies $\omega_{i}$ as

$$
\frac{1}{\pi} \int_{0}^{\omega_{b}} 2 \operatorname{Re}[(\overline{\hat{\boldsymbol{u}}} \cdot \nabla) \hat{\boldsymbol{u}}] d \omega \simeq 2 \sum_{i=1}^{n_{f}} \alpha_{i} 2 \operatorname{Re}\left[\left(\overline{\hat{\boldsymbol{u}}}_{i} \cdot \nabla\right) \hat{\boldsymbol{u}}_{i}\right],
$$

where $\alpha_{i}$ denote appropriate quadrature coefficients and $n_{f}$ represents the number of discrete frequencies. Correspondingly, the amplitude of the response is written as

$$
R^{2}=\left\langle\int_{\Gamma_{\text {in }}} \boldsymbol{u}^{\prime 2} d \Gamma_{\text {in }}\right\rangle \simeq 2 \sum_{i=1}^{n_{f}} \alpha_{i}\left\|\hat{\boldsymbol{u}}_{i}\right\|_{\Omega}^{2}=\frac{2 \pi}{\omega_{b}} \sum_{i=1}^{n_{f}} \alpha_{i} G^{2}\left(\omega_{i}\right) A^{2},
$$

where $A$ is the forcing amplitude previously defined in Eq. (10). The total nonlinear response is then approximated as

$$
\boldsymbol{u}^{\prime} \simeq \sum_{i=1}^{n_{f}} \sqrt{\alpha_{i}}\left(\hat{\boldsymbol{u}}_{i} e^{i\left(\omega_{i} t+\phi_{i}\right)}+\text { c.c. }\right)
$$

where $\phi_{i}$ are unknown phases that remain random. It should be noted that the results of the model (15) are independent of the random phases $\phi_{i}$ since the Reynolds stress (16) is constructed by the product of the response and its complex conjugate, thus canceling the $\phi_{i}$ and $\omega_{i}$ contribution.

To minimize the number $n_{f}$ of discrete frequencies and to approximate the response (17) well enough, we have to select appropriately the discrete frequencies $\omega_{i}$ and weights $\alpha_{i}$, since the gain $G\left(\omega_{i}\right)$ varies strongly with the frequency (Fig. 2). Therefore, we rewrite the nonlinear gain as

$$
G_{\mathrm{tot}}^{2}=\int_{0}^{\omega_{b}} G_{B}^{2}(\omega)\left(\frac{G^{2}(\omega)}{G_{B}^{2}(\omega)}\right) d \omega \simeq \frac{2 \pi}{\omega_{b}} \sum_{i=1}^{n_{f}} \gamma_{i} \frac{G^{2}\left(\omega_{i}\right)}{G_{B}^{2}\left(\omega_{i}\right)},
$$

and we use a weighted Gaussian quadrature rule for $\left(\frac{G^{2}(\omega)}{G_{B}^{2}(\omega)}\right)$ with weight function $G_{B}^{2}(\omega)$ [36]. For a given frequency integration interval, this yields optimal quadrature coefficients $\gamma_{i}$ and abscissas $\omega_{i}$, from which the $\alpha_{i}$ in Eqs. (16) and (17) are easily deduced, $\alpha_{i}=\gamma_{i} / G_{B}^{2}\left(\omega_{i}\right)$. This formulation provides a fast convergence rate when $\left[G^{2}(\omega) / G_{B}^{2}(\omega)\right]$ is close to a constant, in other words when the saturated gain around the mean flow $G(\omega)$ has a similar shape to the linear gain around the base flow $G_{B}(\omega)$.

The Reynolds stress forcing is built by the response structures $\hat{\boldsymbol{u}}_{i}$ multiplied by their corresponding gains $G\left(\omega_{i}\right)$. This approximation strongly depends on the selected discrete frequencies $\omega_{i}$, since in addition to the strong variation of the gain $G\left(\omega_{i}\right)$, the response structure $\hat{\boldsymbol{u}}_{i}$ significantly depends on the frequency, as illustrated clearly in Fig. 2 for the linear case. The frequencies should therefore be selected clustered around the optimal gain $\omega_{\text {opt }} / 2 \pi=0.075$ while spread enough to ensure a rich family of response structures $\hat{\boldsymbol{u}}_{i}$ from which the Reynolds stress forcing is calculated. A suitable distribution is achieved by selecting the optimal frequency as the end point for a Gauss-Radau quadrature rule, which is applied to the two intervals that appear at the right and left side of the optimal frequency. Referring in anticipation to Fig. 7 and looking at the evolution of the overall gain $G_{\text {tot }}$ with $n_{f}$ (squares), we see that this scheme converges very quickly, achieving convergence for a very small number of discrete frequencies $n_{f} \simeq 10$.

It should be highlighted that the coupled system has to be solved iteratively, in order to obtain the correct mean flow $\boldsymbol{U}$ and responses $\boldsymbol{u}_{i}$ for a given forcing $\boldsymbol{f}$ that couples the system. The details on the procedure undertaken to solve the self-consistent system (15) are described in Ref. [19] for a system with harmonic forcing. The unique difference with the present case is that the linear responses at each frequency have to be computed together to obtain the total Reynolds stress forcing in Eq. (15a). Nevertheless, note that the process is parallelizable since all the linear equations are decoupled and are computed independently, in contrast to what happens in a full DNS where all frequencies are 

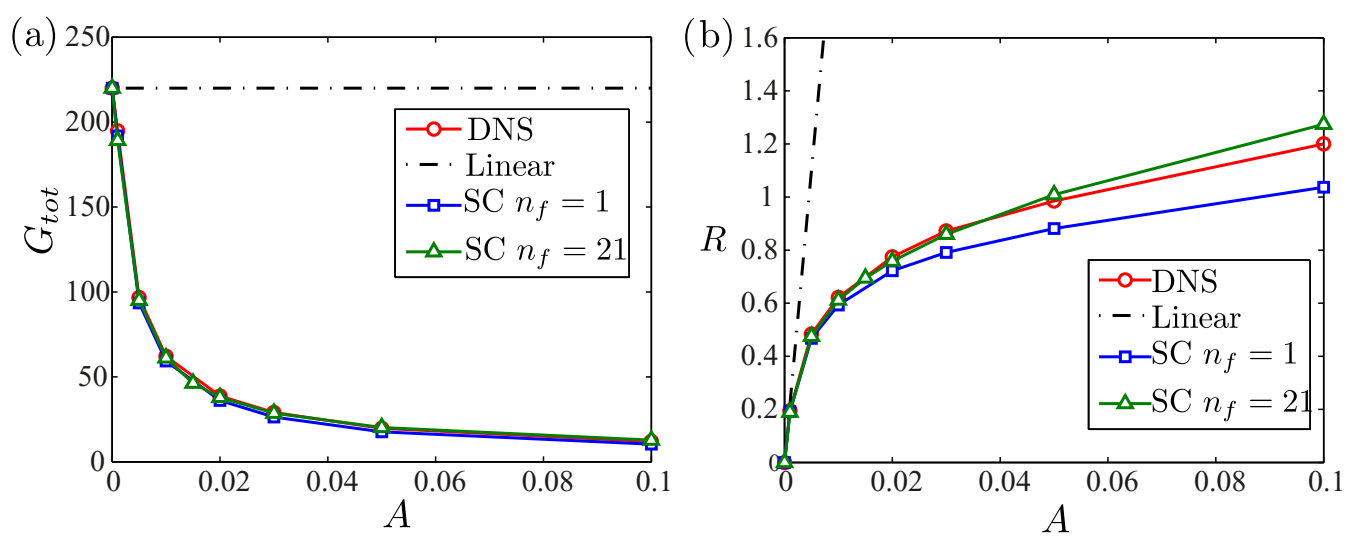

FIG. 6. (a) Gain and (b) response as a function of the forcing amplitude $A$ of the band limited white noise for the DNS (circles), SC model with $n_{f}=21$ (triangles), SC model with $n_{f}=1$ at $\omega_{\text {opt }}$ (squares), and the linear estimation (dash dotted line). $\mathrm{Re}=500$.

coupled due to the term on the right-hand side of (14b). This implies that the computational time for the present model is mainly independent of $n_{f}$ provided there is a correct parallelization.

The introduced approximation scheme (19) can be pushed to the limit $n_{f}=1$ by approximating the nonlinear gain $G(\omega)$ just with a single abscissa point at $\omega_{\text {opt }}$. In this case, the nonlinear gain $G(\omega)$ at the rest of the frequencies is obtained from the weighting function $G_{B}(\omega)$ (see Fig. 2) scaled with the saturated gain around the mean flow $G\left(\omega_{\text {opt }}\right)$ at the optimal frequency $\omega_{\text {opt }}: G^{2}(\omega) \sim \kappa G_{B}^{2}(\omega)$ with $\kappa=G^{2}\left(\omega_{\text {opt }}\right) / G_{B}^{2}\left(\omega_{\text {opt }}\right)$.

The system (15) models the nonlinear behavior of the flow as a response to stochastic forcing. It is an extension of the SC model recently introduced for harmonic forcing [19], and it appears as the forced counterpart of the self-consistent model introduced first for unstable flows $[32,33]$ where the linear equation corresponds to an eigenvalue problem and the amplitude is dictated by the marginal stability criterion.

\section{Results: Dynamics of the flow subject to temporal stochastic forcing}

Applying the SC model to the backward-facing step with increasing forcing amplitude $A$, the model is seen to capture accurately the saturation behavior with a remarkably accurate prediction of the gain and response amplitude as illustrated in Fig. 6, where the DNS results are compared to the SC model. The SC model exhibits a slightly better prediction for $n_{f}=21$ than for a unique frequency $\omega_{\text {opt }}$ approximation of the Reynolds stress $n_{f}=1$, where the construction of the Reynolds stress is far more restrictive, since it is built solely on the information pertaining to the response field at the optimal frequency.

These results suggest that the findings obtained for harmonic forcing [19] extrapolate to white noise forcing. Therefore, Fig. 6 confirms a picture where the mean flow modification due to the nonlinear forcing of the Reynolds stress is crucial to capture the energy saturation for the flow under white noise forcing. Additionally, the nonlinear interaction of the response fluctuation with itself gathered in the term $-\left(\boldsymbol{u}^{\prime} \cdot \nabla\right) \boldsymbol{u}^{\prime}+\left\langle\left(\boldsymbol{u}^{\prime} \cdot \nabla\right) \boldsymbol{u}^{\prime}\right\rangle$ does not seem to play a relevant role in the saturation and can be neglected as assumed in the SC model. In other words, a larger forcing entails a larger response, which in turn generates stronger Reynolds stresses that force and modify the mean flow enforcing a saturation, which reduces the response in comparison to its linear prediction.

Restricting our attention to a saturated case for a forcing amplitude $A=0.1$, Fig. 7 presents the variation of the total gain of the self-consistent model with a different number $n_{f}$ of discrete frequencies $\omega_{i}$ showing a clear increase in accuracy with an increase in the number of points $n_{f}$. It shows that $n_{f}$ as small as 10 is enough to obtain an accurate estimation of the total gain. Note that the difference between the DNS gain and the SC gain does not tend to zero as $n_{f}$ increases, probably due 


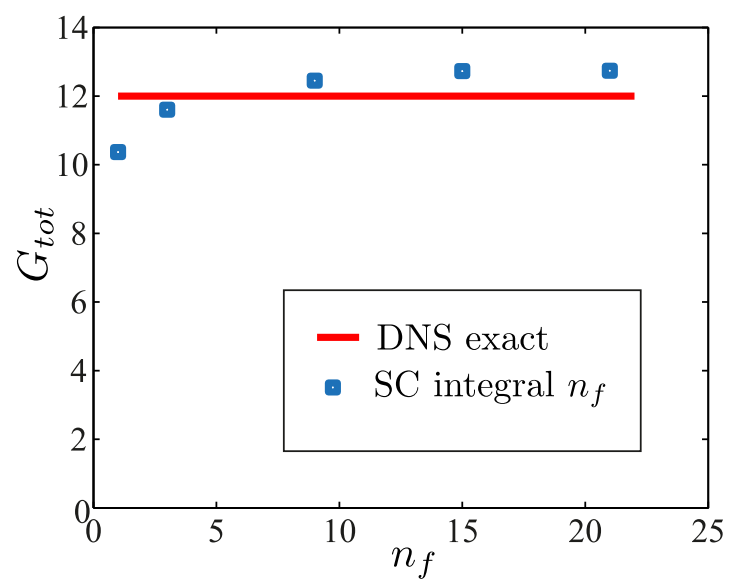

FIG. 7. Total gain of the exact DNS (solid line) and SC model as a function of the number of discrete frequencies $\omega_{i}$ given by $n_{f}$ for the saturated mean flow with a forcing amplitude $A=0.1$ and $\operatorname{Re}=500$.

to the inherent assumptions of the model, which by construction is neglecting the coupling between the different frequencies represented on the right-hand side of (14b). Nonetheless, the SC model presents an estimation of the exact DNS gain in the same order of magnitude, around $G_{\text {tot }}=12$ with a relative difference of less than $10 \%$, which is much smaller than the base flow linear prediction of $G_{\text {tot }}=220$ with a relative difference of more than $1500 \%$.

Figure 8(a) depicts the gain as a function of frequency for the DNS and SC model. The linear response computed around the DNS mean flow (thin solid line) predicts an amplitude and gain $G_{\text {tot }}=9.7$ close to the exact DNS $G_{\text {tot }}=12$, when compared to the linear gain around the base flow $G_{\text {tot }}=220$. It therefore qualitatively, if not fully quantitatively, captures the nonlinear saturation. This result is in line with the linear response to harmonic forcing around the mean flow presented in Ref. [19] and with the linear stability analysis around the mean flow for the cylinder case, which provides an accurate estimation of the frequency and structure of the nonlinear fluctuations [29].
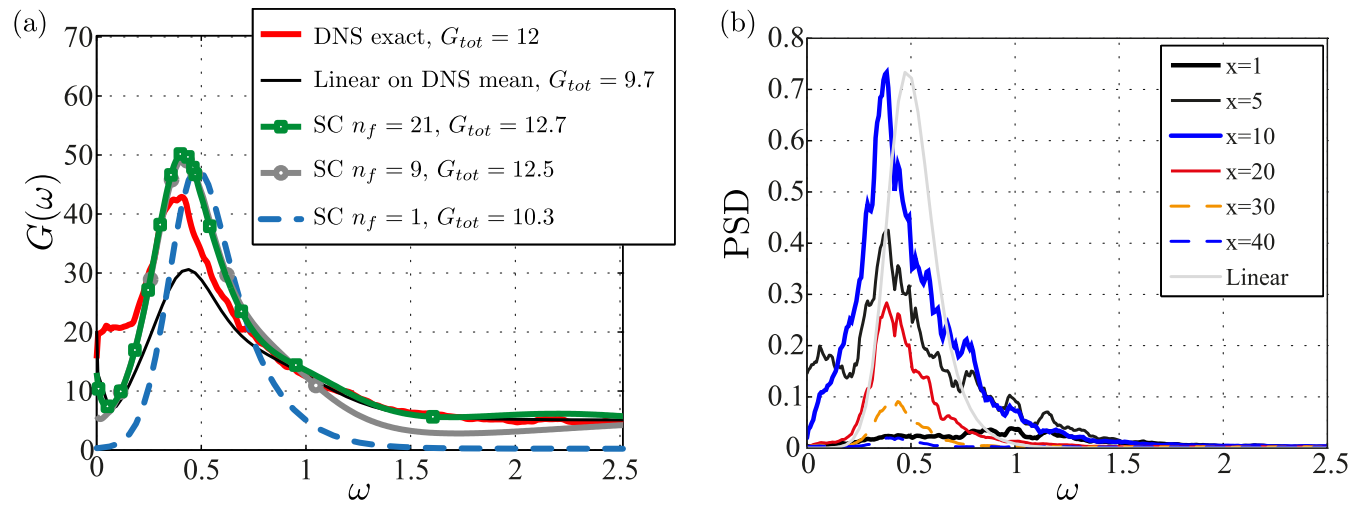

FIG. 8. (a) Gain distribution function of the frequency and total gain values for the DNS and SC model saturated with a forcing amplitude $A=0.1$. The exact DNS total gain is $G_{\text {tot }}=12$ (thick solid line) and $G_{\text {tot }}=9.7$ for the linear prediction around the DNS mean flow (thin solid line). The SC model integrated in frequency has a total gain $G_{\mathrm{tot}}=12.7$ (squares) for $n_{f}=21, G_{\mathrm{tot}}=12.5$ for $n_{f}=9$ (circles), and $G_{\mathrm{tot}}=10.3$ for $n_{f}=1$ (thick dashed line). (b) PSD as a function of the frequency for different positions $x$ at the centerline, $(y=0)$, for the DNS fluctuating velocity field for $A=0.1$ and $\operatorname{Re}=500$. 
The SC model with $n_{f}=21$ and 9 marked with squares and circles shows an acceptable prediction of the exact DNS gain distribution marked as a continuous thick line, presenting a peaked maximum very close to the DNS most amplified frequency but slightly smaller and differing only at very low frequencies. The exact DNS gain distribution is computed from a PSD of the time varying simulation at different points and then integrated over the whole domain $\Omega$ at each frequency $\omega$. The gain distribution of the base flow scaled by the gain at the optimal frequency of the SC model at $G\left(\omega_{\text {opt }}\right)$ (thick dashed line) does not approximate well the exact DNS distribution, although it is used to weight the response and the Reynolds stress for $n_{f}=1$ in Eq. (19) and estimates correctly the saturation (Fig. 7) with an integral in frequency $G_{\text {tot }}=10.3$ rather close to the exact DNS one.

Note that the nonlinear interactions in the saturation process entail not only a reduction in the total gain but also a slight shift in the optimal frequency as illustrated in Fig. 8(a) when comparing the base flow gain scaled with the DNS results. Nevertheless, this shift is small enough for the single frequency approximation $n_{f}=1$ to capture the nonlinear saturation in terms of its energy despite being fixed at the base flow optimal frequency $\omega_{\text {opt }}$.

Figure 8(b) presents the PSD of the DNS response to forcing with amplitude $A=0.1$ at different positions $x$ along the centerline of the domain $(y=0)$ where the response is strong. An interesting feature is that the dominant frequency is constant at all positions, showing that there is not any zone with a strong competition between oscillating responses at different frequencies and hence the whole response oscillates mainly as a unique coherent structure.

The nonlinear total gain is reminiscent of the idea of a nonlinear transfer function, with a gain at each frequency $G(\omega)$ that depends on the amplitude of the forcing and the frequency. The concepts of a nonlinear transfer function and nonlinear gain are well described by Noiray [37] dealing with the study of nonlinear stability of flames in burners.

The local kinetic energy and the velocity in the $x$-direction of the response fluctuation are compared in Fig. 9 for the linear approximation around the base flow, the DNS, and the SC model. The energy distribution of the SC model with $n_{f}=21$ [Fig. 9(c)] approximates the exact DNS [Fig. 9(b)] capturing most of the upstream migration when compared to the response structure of the linear estimation around the base flow [Fig. 9(a)]. Nonetheless, the SC model exhibits a more elongated structure compared to the compact DNS energy distribution located between the two recirculation bubbles. The inability of the model to predict the exact response structure is probably caused by the neglected frequency coupling in the model [right-hand side of (14b)], which is present in the full DNS, and the discrete number of harmonics. As one might expect, the SC model with a single frequency approximation of the response $\left(n_{f}=1\right)$, depicted in Fig. 9(d), presents a poorer approximation than its frequency integrated counterpart [Fig. 9(c)].

The DNS snapshot of the fluctuating velocity $\boldsymbol{u}_{x}^{\prime}$ shows in Fig. 9(f) a very clear streamwise wavelength. This means that despite the response being composed of a mix of streamwise wavelengths excited at all frequencies inside the band $\omega_{b}$ (Fig. 2), there is a clear selective process governed by the gain curve of [Fig. 8(b)], which promotes the same optimal frequency and response structure with a constant wavelength in the whole domain. This selective process is well captured by the SC model results, especially by the multifrequency approximation [Fig. 9(g)] that approximates well the streamwise wavelength when compared to the DNS snapshot [Fig. 9(f)].

A more quantitative comparison of the migration upstream of the response with an increase of the amplitude of the forcing illustrated in Fig. 5 is described by the position of the maximum $x_{E \max }$ of the fluctuation energy and plotted in Fig. 10. Although the SC model estimates very closely the trend of the DNS response migration, there is a constant difference of $\delta x_{E \max } \sim 3 h$, which is slightly larger for the single frequency approximation (triangles) and is in line with the results shown in Fig. 9. It should be noted that the SC model is able to describe more accurately the response saturation in terms of amplitude, Fig. 6, than of structure and position, possibility because the response amplitude is an integral quantity and because of the missing cross-coupling between frequencies in the SC model.

Focusing on the mean flow modification, Fig. 11 compares the DNS mean flow $\boldsymbol{U}$ for a forcing amplitude $A=0.1$ and the base flow $\boldsymbol{U}_{B}$, thus highlighting the shortening of the bottom recirculation 
(a) Linear Base

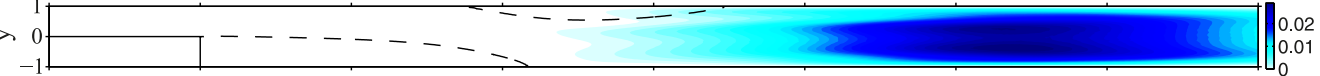

(b)

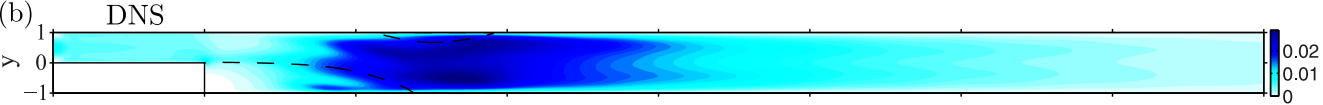

(c) $\mathrm{SC} n_{f}=21$

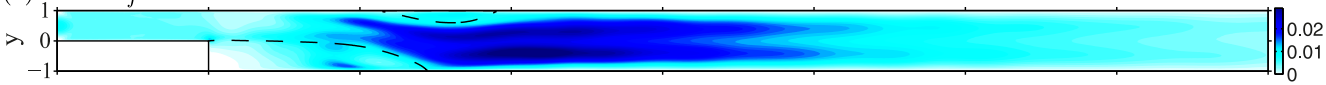

(d) ${ }_{1}$ SC $f=0.075$
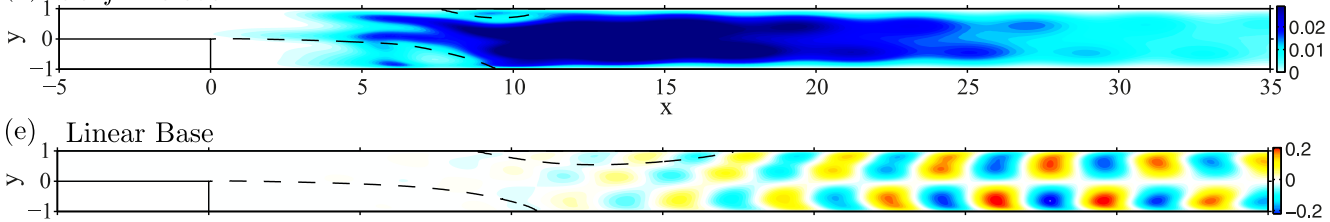

(f) DNS
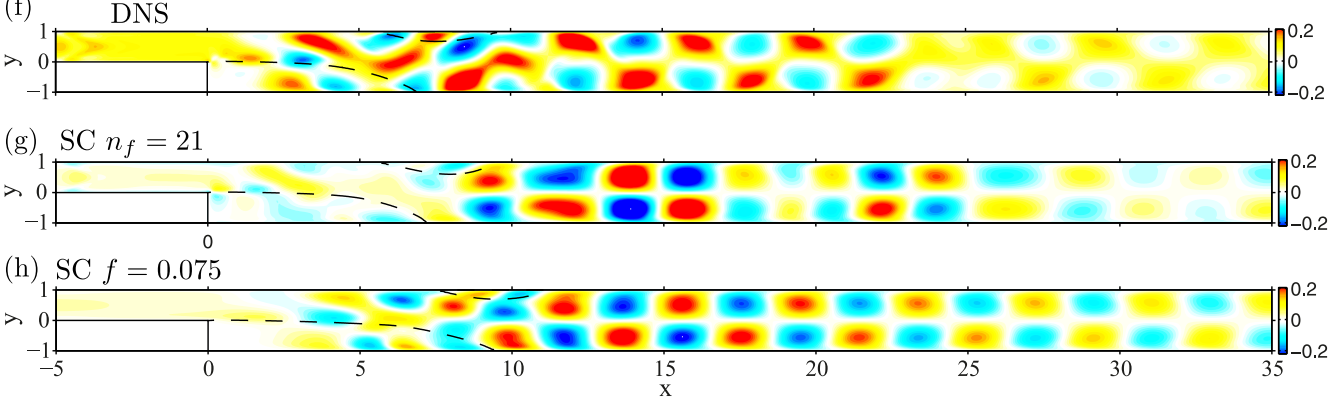

FIG. 9. Energy distribution of the response fluctuation for (a) linear around base flow, (b) DNS, (c) SC with $n_{f}=21$, and (d) SC with single frequency approximation $\omega_{\mathrm{opt}}$. Fluctuation velocity in the $x$-direction, $u_{x}^{\prime}$ for (e) linear around base flow, (f) DNS snapshot, (g) SC with $n_{f}=21$ constructed with arbitrary $\phi_{i}$ in Eq. (18), and (h) SC with $n_{f}=1$, single frequency approximation at $\omega_{\text {opt }}$. Forcing amplitude $A=0.1$ and $\operatorname{Re}=500$.

bubble and the upstream migration of the top recirculation bubble. In addition, Figs. 11(c) and 11(d) illustrate the difference between the mean flow and base flow defined as the mean flow correction in Eq. (13) and showing that the SC model estimates well the DNS mean flow.

A quantitative analysis of the mean-field modification is presented in Fig. 12 where the mean flow recirculation bubble lengths are compared for the DNS and SC model as a function of the forcing amplitude. The SC model with $n_{f}=21$ (circles) predicts accurately the bubble positions, for the top as much as for the bottom, even capturing very closely the nonmonotonous trend of the bubble at the bottom. This is of great relevance because it implies that the SC model is able to characterize properly the flow where linear estimations around the base flow would fail. The SC model with $n_{f}=1$ (triangles) also follows the migration of the bubbles of the DNS solution (squares), although not as closely as the integrated form mainly describing the nonmonotonous behavior. This difference appears probably due to the restricted construction of the Reynolds stress forcing by the only optimal response structure.

\section{SPATIOTEMPORAL STOCHASTIC FORCING}

\section{A. Formulation}

Since realistic external disturbances entering in real flows do not generally present a coherent spatial distribution, the study is now generalized by imposing a white noise forcing uncorrelated in 


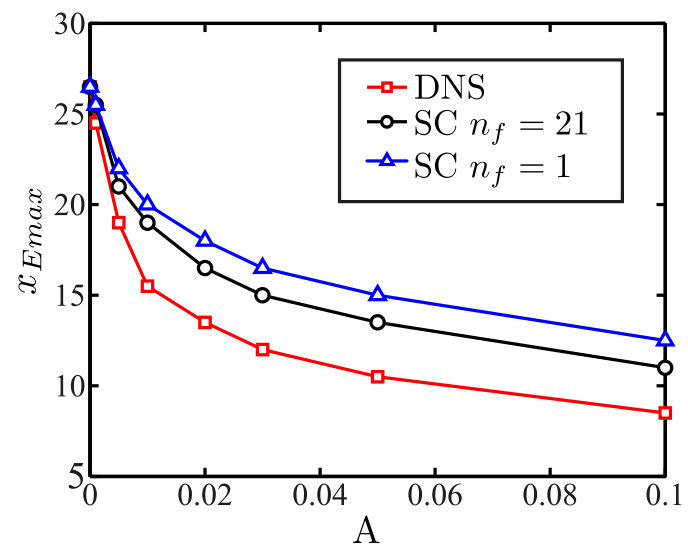

FIG. 10. Position in the $x$ coordinate of the maximum of the energy of the perturbation $x_{\max }$ for SC and DNS as a function of the forcing amplitude $A$ for $\operatorname{Re}=500$.

space and time, $\boldsymbol{f}(y, t)=A \boldsymbol{\xi}(y, t)$ at the inlet [17,23]. The stochastic vector $\boldsymbol{\xi}(y, t)$ is a Gaussian random process that represents the band-limited white noise, which is $\delta$-correlated in space and time, has zero mean, and has unit variance. Discretizing the section $\Gamma_{\text {in }}$ in $n_{k}$ degrees of freedom, $\boldsymbol{\xi}(y, t)$ becomes a column vector of $n_{k}$ random variables, and $\boldsymbol{\xi}(y, t)$ is normalized in such a way that the power of $\boldsymbol{f}$ is $A^{2}$.

Instead of using the finite element basis to represent the spatial noise distribution, it is convenient to use the orthogonal basis resulting from the SVD analysis of the resolvent operator $\mathscr{R}$. Introducing the adjoint of the resolvent operator $\mathscr{R}^{\dagger}$, the linear gain can indeed be rewritten as a Rayleigh quotient of the resolvent operator and the forcing,

$$
G_{1}^{2}(\omega)=\max _{\tilde{f}} \frac{(\mathscr{R} \tilde{\boldsymbol{f}} \mid \mathscr{R} \tilde{\boldsymbol{f}})}{(\tilde{\boldsymbol{f}} \mid \tilde{\boldsymbol{f}})}=\max _{\tilde{f}} \frac{\left(\mathscr{R}^{\dagger} \mathscr{R} \tilde{\boldsymbol{f}} \mid \tilde{\boldsymbol{f}}\right)}{(\tilde{\boldsymbol{f}} \mid \tilde{\boldsymbol{f}})} .
$$

Subsequently, the optimal gain and forcing on the base flow $\boldsymbol{U}_{B}$ correspond to the leading eigenvalue $\lambda_{1}=G_{1}^{2}$ and eigenvector $\tilde{\boldsymbol{f}}_{1}$ of the symmetrical eigenvalue problem $\mathscr{R}^{\dagger} \mathscr{R} \tilde{\boldsymbol{f}}_{k}=\lambda_{k} \tilde{\boldsymbol{f}}_{k}$ computed at

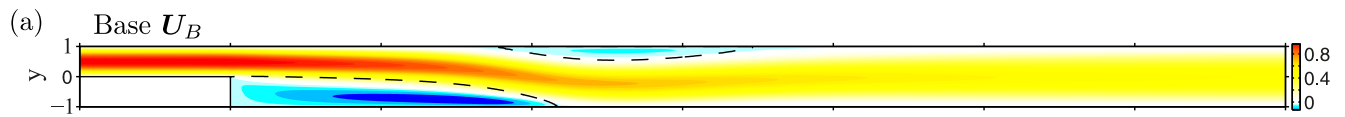

(b) Mean DNS $\boldsymbol{U}$
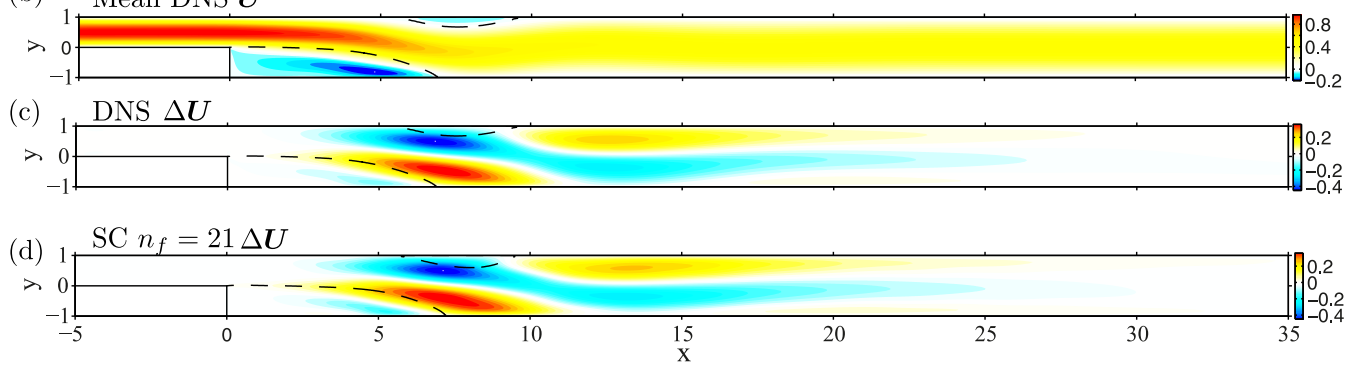

FIG. 11. Comparison of the mean flow and base flow for the velocity in the $x$-direction: (a) base flow $\boldsymbol{U}_{B}$ and (b) DNS mean flow $\boldsymbol{U}$. Difference between the mean flow and the base flow $\Delta \boldsymbol{U}$ defined as mean flow correction in Eq. (13), (c) $\Delta \boldsymbol{U}$ for the DNS mean flow, and (d) $\Delta \boldsymbol{U}$ for the SC mean flow for $n_{f}=21$. Forcing amplitude $A=0.1$ and $\operatorname{Re}=500$. 

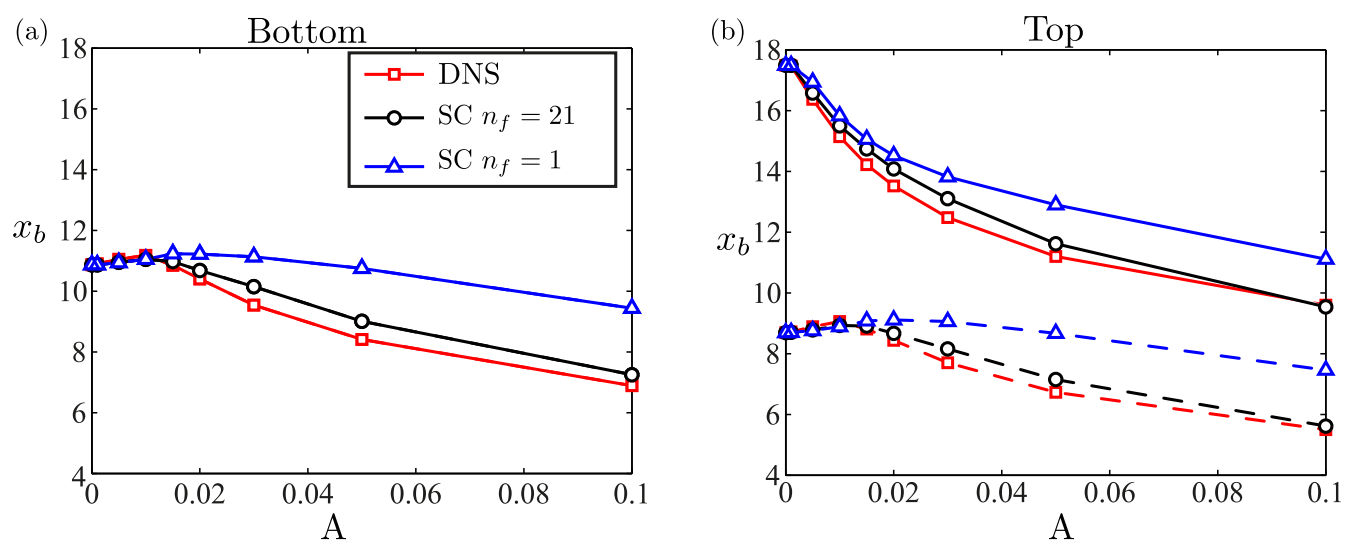

FIG. 12. Position of the mean flow recirculation bubbles (a) bottom and (b) top for the DNS (squares), SC integrated in frequency (circles), and SC with optimal frequency approximation (triangles) as a function of the forcing amplitude $A$. $\operatorname{Re}=500$.

any frequency $\omega_{i}$

$$
G_{1}^{2}\left(\omega_{i}\right)=\frac{\left(\mathscr{R}^{\dagger} \mathscr{R} \hat{\boldsymbol{f}}_{i, 1} \mid \hat{\boldsymbol{f}}_{i, 1}\right)}{\left(\hat{\boldsymbol{f}}_{i, 1} \mid \hat{\boldsymbol{f}}_{i, 1}\right)} .
$$

The family of eigenmodes and eigenvalues constitutes a spatial orthogonal basis for the forcing $\tilde{\boldsymbol{f}}_{k}=\hat{\boldsymbol{f}}_{i, k}$ and corresponding response $\hat{\boldsymbol{u}}_{i, k}$ for each frequency $\omega_{i}$ sorted by their associated gain $G_{k}\left(\omega_{i}\right)$ as $G_{1}\left(\omega_{i}\right)>G_{2}\left(\omega_{i}\right)>G_{3}\left(\omega_{i}\right) \ldots$. Since the different structures of optimal and suboptimal forcing are equally energetic, they can be normalized such that $\left\|\hat{\boldsymbol{f}}_{i, k}\right\|_{\Gamma_{\mathrm{in}}}=\pi / n_{k} \omega_{b}$. Hence, the amplitude of the forcing comes as

$$
\left\langle\int_{\Gamma_{\mathrm{in}}} \boldsymbol{f}^{2} d \Gamma_{\mathrm{in}}\right\rangle=\sum_{i=1}^{n_{f}} \alpha_{i} \sum_{k=1}^{n_{k}} 2 A^{2}\left\|\hat{\boldsymbol{f}}_{i, k}\right\|_{\Gamma_{\mathrm{in}}}^{2}=A^{2} .
$$

The total gain corresponds to an integral in the frequency domain and accounts at each frequency for the different possible response structures with their corresponding gains, all of them forced with equally energetic forcing. In analogy to (19), the total gain is written as

$$
G_{\mathrm{tot}}^{2} \simeq \frac{2 \pi}{\omega_{b}} \sum_{i=1}^{n_{f}} \alpha_{i} \frac{1}{n_{k}} \sum_{k=1}^{n_{k}} G_{k}\left(\omega_{i}\right)^{2}
$$

where $\alpha_{i}$ are quadrature coefficients previously introduced, accounting for the discrete frequency distribution.

Truncating the flow dynamics to its most energetic patterns is common in turbulence studies [38]. Thereby, we reduce the complexity of modeling a whole spatiotemporal stochastic forcing by extracting the most amplified structures of the response $\hat{\boldsymbol{u}}_{i, 1}$ with their corresponding forcing $\hat{\boldsymbol{f}}_{i, 1}$ at each frequency in a set of selected frequencies $\omega_{i}$. Note that at each frequency $\omega_{i}$, the optimal gain $G_{1}\left(\omega_{i}\right)$ retrieves the most amplified structures. Hence, knowing that the suboptimal gains are orders of magnitude lower $[17,18]$, we approximate the full response by the most amplified one. The total gain is approximated as

$$
G_{\mathrm{tot}}^{2} \simeq \frac{2 \pi}{\omega_{b}} \sum_{i=1}^{n_{f}} \alpha_{i} \frac{1}{n_{k}}\left(\sum_{k=1}^{n_{k}} \frac{G_{B, k}^{2}\left(\omega_{i}\right)}{G_{B, 1}^{2}\left(\omega_{i}\right)}\right) G_{1}^{2}\left(\omega_{i}\right)=\frac{2 \pi}{\omega_{b}} \sum_{i=1}^{n_{f}} \alpha_{i} \frac{\beta_{i}}{n_{k}} G_{1}^{2}\left(\omega_{i}\right),
$$


where energy weights $\beta_{i}=\sum_{k=1}^{n_{k}} \frac{G_{B, k}^{2}\left(\omega_{i}\right)}{G_{B, 1}^{2}\left(\omega_{i}\right)}$ are calculated at the base flow, where they are known in order to obtain the relation of gains and assuming a constant ratio of the suboptima along the saturation. The coefficients $\beta_{i}$ account for the spatial distribution at each frequency $\omega_{i}$. This procedure recalls the work by Dergham et al. [17], where a low rank approximation of the covariance matrix is built by the most energetic responses integrated discretely in the frequency domain. The amplitude of the response is then approximated as

$$
R^{2} \simeq \sum_{i=1}^{n_{f}} \alpha_{i} \beta_{i} 2\left\|\hat{\boldsymbol{u}}_{i, 1}\right\|_{\Omega}^{2},
$$

and the coupled system of equations of the self-consistent model is rewritten as

$$
\begin{aligned}
\mathscr{N}(\boldsymbol{U}) & =-\sum_{i=1}^{n_{f}} \alpha_{i} \beta_{i} 2 \operatorname{Re}\left[\left(\overline{\hat{\boldsymbol{u}}}_{i, 1} \cdot \nabla\right) \hat{\boldsymbol{u}}_{i, 1}\right], \\
\boldsymbol{U} & =\boldsymbol{u}_{\text {Pois }} \text { on } \Gamma_{\text {in }}, \\
i \omega_{i} \hat{\boldsymbol{u}}_{i, 1}+\mathscr{L}_{\boldsymbol{U}}\left(\hat{\boldsymbol{u}}_{i, 1}\right) & =0, \\
\hat{\boldsymbol{u}}_{i, 1} & =\hat{\boldsymbol{f}}_{i, 1} \text { on } \Gamma_{\text {in }}, \quad \hat{\boldsymbol{f}}_{i, 1} \text { from }(21) .
\end{aligned}
$$

In general, $n_{k}$ depends on the spatial number of the degrees of freedom (NDOF) of the white noise, for our simulations $n_{k}=80$ coinciding with the NDOF of the mesh at the inlet $\Gamma_{\text {in }}$. Changing the underlying mesh while maintaining the $n_{k}$ number yields the same total gain $G_{\text {tot }}$, thus showing that $n_{k}$ is equivalent to the frequency band limit but in space. It represents the band limit of the spatial distribution of the noise, limiting it to a restricted base of $n_{k}$ degrees of freedom. Similarly to what happens in the frequency domain in Eq. (12), increasing the number $n_{k}$ decreases the total gain because energy is spent in finer spatial structures with higher wave number that do not provide large responses.

\section{B. Results: Dynamics of the flow subject to spatiotemporal stochastic forcing}

The self-consistent model is applied to the backward-facing step problem forced at the inlet $\Gamma_{\text {in }}$ with white noise $\delta$-correlated in space and time, with an increasing amplitude $A$ at $\operatorname{Re}=700$. At $\operatorname{Re}=500$ the amplification is very small given a fine enough spatial resolution at the inlet $\Gamma_{\text {in }}$,

(a)

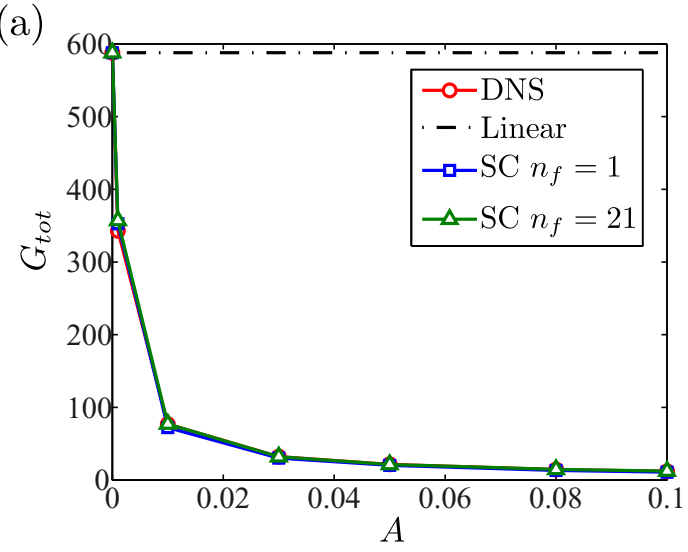

(b)

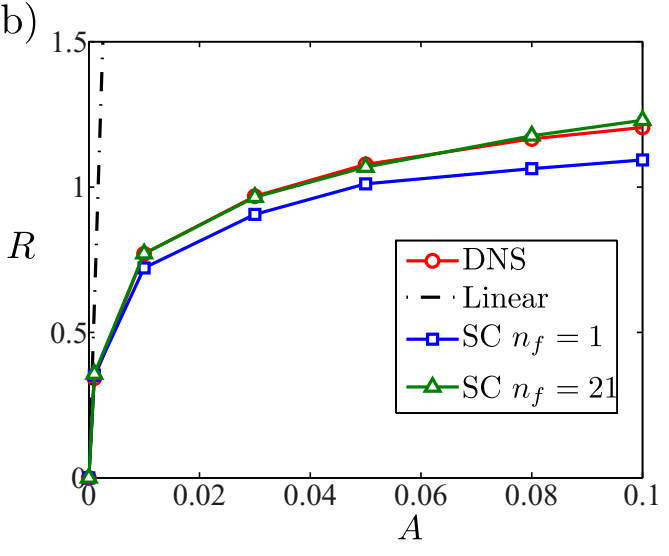

FIG. 13. (a) Gain and (b) response as functions of the forcing amplitude $A$ of the band-limited white noise for the DNS (circles), SC model integral in frequency with $n_{f}=21$ (triangles), SC model with $n_{f}=1$ at $\omega_{\text {opt }}$ (squares), and the linear estimation (dash-dotted line). $\operatorname{Re}=700$. 

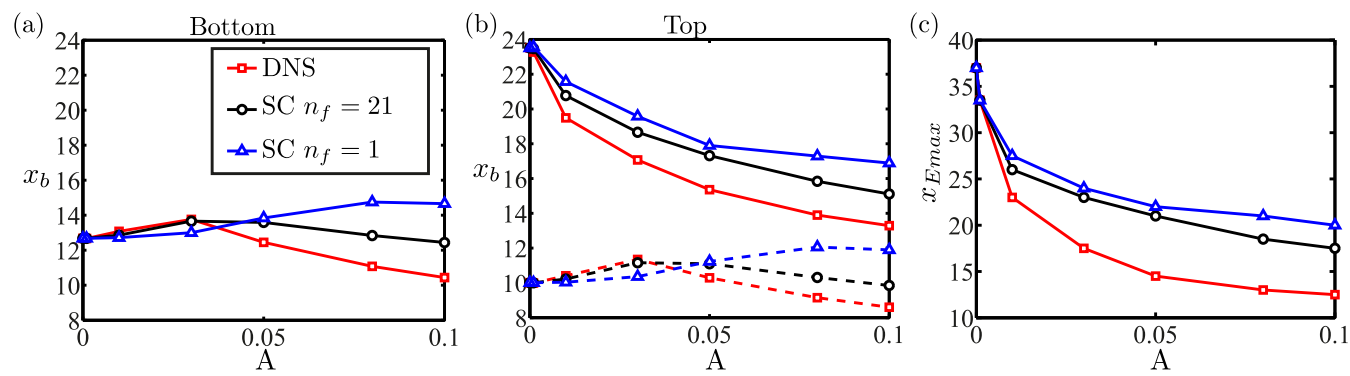

FIG. 14. Position of the recirculation bubbles (a) bottom and (b) top for the DNS (squares), SC model integrated in frequency (circles), and SC model with optimal frequency approximation (triangles) as a function of the forcing amplitude $A$. (c) Position maximum of the energy of the perturbation $x_{E \max }$ for the SC model and DNS as a function of the forcing amplitude $A$. $\operatorname{Re}=700$.

therefore, to obtain a larger amplification, we increased the Reynolds number to $\operatorname{Re}=700$ since the gain increases rapidly with the Reynolds number [18]. The SC model estimates distinctively well the saturation of the gain and response as illustrated in Fig. 13. The SC with $n_{f}=1$ presents a slightly lower response when compared to DNS, but a minimal difference when compared to the linear prediction, which is incorrect in orders of magnitude for a strong saturation. It should be highlighted that the Reynolds stress coupling in the self-consistent model allows us to capture the nonlinear response to white noise approximating the whole stochastic response that varies in space and time with the structure of the response solely at the optimal frequency.

A more quantitative comparison of the flow features between the SC model and the exact DNS reveals that the SC model captures the main trends in the variation of the flow configuration as presented in Fig. 14, however not as accurately as the estimation of the global energy of the response. Focusing on the recirculation bubbles of the mean flow, Figs. 14(a) and 14(b), the SC model with $n_{f}=21$ follows approximately the DNS capturing the nonmonotonous behavior. Nevertheless, the SC model with a single frequency approximation $n_{f}=1$ provides only a very coarse estimation of the recirculation bubble migration and fails to capture the nonmonotonous trend. In terms of the position of the maximum of the fluctuating energy, the SC model captures well the migration upstream but maintains a mismatch for large saturations as depicted in Fig. 14(c). As one could expect, this mismatch is stronger for the SC model for $n_{f}=1$ than for $n_{f}=21$. It should be noted that the self-consistent model is able to well approximate the flow behavior given the strong assumptions involved, consolidating the Reynolds stress as the key nonlinear term implicated in the saturation process.

\section{DISCUSSION AND CONCLUSIONS}

The backward-facing step is a well known example of an amplifier in which small perturbations to the steady base state undergo large amplifications due to the non-normality of the linearized equations. These amplifications depend strongly on the frequency of the perturbations $[15,18]$ showing a low frequency band pass filtering with an optimal frequency. In the present work, the backward-facing step is forced at the inlet with a band-limited white noise to model disturbances that appear naturally in flows. We apply a self-consistent model to describe the nonlinear dynamics of the filtering and the saturation of the response with an increasing amplitude of the stochastic forcing.

The study of the stochastic forcing and response is addressed rewriting the self-consistent model in the frequency domain. It consists in a coupled system of the mean flow equation and the linear response equation around the aforementioned mean flow at a finite number of discrete frequencies (typically in the order of 10). The coupling is attained by the Reynolds stress that forces the mean flow and is constructed from the integration of the different responses in the frequency domain 
using a proper energy weight. The model is applied to the nonlinear saturation problem, starting with a forcing with a given spatial structure but stochastic in time, obtaining a remarkably accurate prediction of the global saturation of the response when compared to the DNS results. The approach captures the small shift in the dominant frequency of the nonlinear response with respect to the linear response. An interesting feature of the response is that it presents the same dominant frequency at different streamwise stations and is thus governed mainly by a single coherent structure oscillating at the preferred frequency. This allows us to push the model to a single frequency approximation of the stochastic response. A more quantitative comparison describes an acceptable estimation of the shortening of the mean recirculation bubble capturing the nonmonotonic trend. In addition, the comparison of the fluctuating response shows that the model predicts well the upstream migration of the response, while providing an estimation of the streamwise wavelength despite the stochasticity of the response.

The response to a more realistic disturbance, described by a stochastic forcing $\delta$-correlated in space and time, is modeled by extracting the most energetic structures [17] as commonly used in turbulence studies [38]. Based on these optimal structures, the SC model is rewritten using the optimal forcing and corresponding response at each frequency with their proper energetic weights. The reformulated model is able to estimate accurately the saturation of the response as well as the decrease of the mean recirculation bubble and the upstream migration of the response, despite the stochasticity of the forcing.

The results obtained suggest that the nonlinear dynamics of the saturation process in the backwardfacing step is mainly governed by the nonlinear Reynolds stress forcing even for a stochastically forced flow, and that the nonlinear interaction of the fluctuation with itself has a secondary effect. Besides, it should be noted that the full response to stochastic noise is well approximated by the most energetic structure at the optimal frequency. The presented model follows as an extension to stochastic flows of the self-consistent model proposed for harmonic fluctuations. It was applied to an amplifier to calculate the saturation of the response to harmonic forcing on the backward-facing step [19], and to an oscillator, the unstable cylinder wake [32,33], where the instability is dominated by the most unstable eigenmode, and its saturation is dictated by the unstable eigenmode marginality criterion $[29,39,40]$. Therefore, a common physical picture is revealed: as the fluctuations grow due to an increasing response to forcing or an instability mechanism, respectively, it creates a Reynolds stress forcing that modifies the mean flow reducing its amplification and thus saturating the flow response.

One of the fundamental aspects behind the self-consistent model is that the full nonlinear response to stochastic forcing is approximated by a linear response at different forcing frequencies. Still, the model is able to estimate the nonlinear transfer function of the system, and to approximate the nonlinear filtering from the input flat white noise forcing into a band pass frequency response. It should be highlighted that the solutions of the self-consistent model are obtained a priori without using any DNS or experimental data. Similar models where the mean flow is coupled to the linear perturbation equations through the Reynolds stress forcing can be found in the study of turbulent flows, as, for example, computing coherent structures that appear during the transition in a parallel shear flow [26] or by restricted nonlinear dynamics (RNL) of DNS with a linear fluctuation equation [25]. Another example is the stochastic structural stability theory (SSST) [22,23] where the ensemble average mean flow equation is coupled to the linear response formulated in terms of the covariance matrix and governed by the Lyapunov equation. The SSST has been applied to parallel turbulent flows describing well the nonlinear behavior that produces sustained coherent structures. Nonetheless, the requirement of solving a Lyapunov equation limits the domain size and geometry due to the computational cost. In this sense, a low order approximation to the covariance matrix has been proposed to approximate the linear response [17,21] with respect to which our present model is a limiting case with strong assumptions but is still able to capture the main nonlinear behavior of the system.

Furthermore, the SSST and RNL are restricted to parallel flows to obtain the ensemble average from the $x$-direction average, while our model does not present any geometry constriction. However, 
these models are able to capture the transition dynamics while the SC model as presented is restricted to the saturated state. In addition, the RNL model is time integrated, thus it accounts for all the harmonics until the cutoff (time step dependent), while the present SC model shows the frequency selective nature of the response allowing to capture the major part of the saturation process with only a few harmonics.

The present SC model, as well as SSST [22,23], RNL [25], and the model in Ref. [26], all rely on the common assumption of eliminating the nonlinearity arising from the cross-coupling between the different frequencies [right-hand side of (14b)] while maintaining solely the nonlinearity of the Reynolds stress retroaction onto the mean flow. This assumption implies that the proposed models are meant for linearly stable flows where self-sustained instabilities are naturally damped. While we cannot exclude noise-induced subcritical transitions even in nominally linearly stable flows, we have not observed such a nonlinear subcritical transition scenario. The presently described mean flows were checked to remain linearly stable. Additionally, for the decoupling of the harmonic components to be a reasonable assumption, there should be limited harmonic generation and subharmonic excitation. In the present flow, this can be interpreted to result from the spatial decorrelation between the response to a considered frequency and the optimal forcings at its harmonic and subharmonic frequencies. However, it should be highlighted that the harmonic interaction is Reynolds and confinement dependent and that increasing the amplitude of the forcing brings the response closer to the inlet, thus approaching it from the more receptive region, suggesting that at very high forcing amplitudes or higher Reynolds number the harmonic interactions may start to become important. Further studies are required to assess the limits and capabilities of the model for flows with higher harmonic generation and subharmonic instability.

These restrictions apply to the SC model on the backward-facing step flow at the studied Reynolds numbers since the flow presents mainly $2 \mathrm{D}$ dynamics [12] and it is stable up to $\operatorname{Re}_{\mathrm{cr}} \sim 748$ [27,28], where a steady $3 \mathrm{D}$ instability appears. Although the model can be extrapolated to 3D flows, one would have to cope with the natural growth and saturation of the static $3 \mathrm{D}$ instability mode and thereby generalize the proposed model.

As a final note, the present model is not conceived as a substitute for the Navier-Stokes equations. On the contrary, its significance lies in the integration of only the essential ingredients required to provide a fairly accurate description of the physics. It remains to be seen whether the present self-consistent model works in other globally stable laminar flows excited by stochastic forcing.

\section{ACKNOWLEDGMENTS}

The authors are very grateful to Tomas Tangarife for stimulating discussions. The authors would also like to acknowledge Saviz Mowlavi for his useful comments.

[1] J.-M. Chomaz, Global instabilities in spatially developing flows: Non-normality and nonlinearity, Annu. Rev. Fluid Mech. 37, 357 (2005).

[2] P. J. Schmid, Nonmodal stability theory, Annu. Rev. Fluid Mech. 39, 129 (2007).

[3] L. N. Trefethen, A. E. Trefethen, S. C. Reddy, and T. A. Driscoll, Hydrodynamic stability without eigenvalues, Science 261, 578 (1993).

[4] B. F. Farrell and P. J. Ioannou, Generalized stability theory. Part 1: Autonomous operators, J. Atmos. Sci. 53, 2025 (1996).

[5] P. J. Schmid and D. S. Henningson, Stability and Transition in Shear Flows (Springer, New York, 2001).

[6] K. M. Butler and B. F. Farrell, Three-dimensional optimal perturbations in viscous shear flow, Phys. Fluids A 4, 1637 (1992).

[7] P. Corbett and A. Bottaro, Optimal perturbations for boundary layers subject to stream-wise pressure gradient, Phys. Fluids 12, 120 (2000). 


\section{VLADISLAV MANTIČ-LUGO AND FRANÇOIS GALLAIRE}

[8] E. Akervik, U. Ehrenstein, F. Gallaire, and D. S. Henningson, Global two-dimensional stability measures of the flat plate boundary-layer flow, Eur. J. Mech. B/Fluids 27, 501 (2008).

[9] A. Monokrousos, E. Akervik, L. Brandt, and D. S. Henningson, Global three-dimensional optimal disturbances in the Blasius boundary-layer flow using time-steppers, J. Fluid Mech. 650, 181 (2010).

[10] D. Sipp and O. Marquet, Characterization of noise amplifiers with global singular modes: The case of the leading-edge flat-plate boundary layer, Theor. Comput. Fluid Dyn. 27, 617 (2013).

[11] F. Alizard, S. Cherubini, and J.-C. Robinet, Sensitivity and optimal forcing response in separated boundary layer flows, Phys. Fluids 21, 064108 (2009).

[12] H. M. Blackburn, D. Barkley, and S. J. Sherwin, Convective instability and transient growth in flow over a backward-facing step, J. Fluid Mech. 603, 271 (2008).

[13] M. R. Jovanović and B. Bamieh, Componentwise energy amplification in channel flows, J. Fluid Mech. 534, 145 (2005).

[14] X. Garnaud, L. Lesshafft, P. J. Schmid, and P. Huerre, The preferred mode of incompressible jets: Linear frequency response analysis, J. Fluid Mech. 716, 189 (2013).

[15] O. Marquet and D. Sipp, Global sustained perturbations in a backward-facing step flow, in Seventh IUTAM Symposium on Laminar-Turbulent Transition (Springer, Netherlands, 2010).

[16] O. Marquet, D. Sipp, and L. Lesshafft, Global stability analysis of open shear flows without global modes, Tech. Rep. (2010).

[17] G. Dergham, D. Sipp, and J.-Ch. Robinet, Stochastic dynamics and model reduction of amplifier flows: The backward facing step flow, J. Fluid Mech. 719, 406 (2013).

[18] E. Boujo and F. Gallaire, Sensitivity and open-loop control of stochastic response in a noise amplifier flow: The backward-facing step, J. Fluid Mech. 762, 361 (2015).

[19] V. Mantič-Lugo and F. Gallaire, Self-consistent model for the saturation mechanism of the response to harmonic forcing in the backward-facing step flow, J. Fluid Mech. 793, 777 (2016).

[20] B. F. Farrell and P. J. Ioannou, Stochastic forcing of the linearized Navier-Stokes equations, Phys. Fluids A 5, 2600 (1993).

[21] B. F. Farrell and P. J. Ioannou, Accurate low-dimensional approximation of the linear dynamics of fluid flow, J. Atmos. Sci. 58, 2771 (2001).

[22] B. F. Farrell and P. J. Ioannou, Structural stability of turbulent jets, J. Atmos. Sci. 60, 2101 (2003).

[23] B. F. Farrell and P. J. Ioannou, Dynamics of streamwise rolls and streaks in turbulent wall-bounded shear flow, J. Fluid Mech. 708, 149 (2012).

[24] F. Bouchet, C. Nardini, and T. Tangarife, Kinetic theory of jet dynamics in the stochastic barotropic and 2D Navier-Stokes equations, J. Stat. Phys. 153, 572 (2013).

[25] V. L. Thomas, B. K. Lieu, M. R. Jovanović, B. F. Farrell, P. J. Ioannou, and D. F. Gayme, Self-sustaining turbulence in a restricted nonlinear model of plane Couette flow, Phys. Fluids 26, 105112 (2014).

[26] C. Beaume, G. P. Chini, K. Julien, and E. Knobloch, Reduced description of exact coherent states in parallel shear flows, Phys. Rev. E 91, 043010 (2015).

[27] D. Barkley, M. G. M. Gomes, and R. D. Henderson, Three-dimensional instability in flow over a backwardfacing step, J. Fluid Mech. 473, 167 (2002).

[28] D. Lanzerstorfer and H. C. Kuhlmann, Three-dimensional instability of the flow over a forward-facing step, J. Fluid Mech. 695, 390 (2012).

[29] D. Barkley, Linear analysis of the cylinder wake mean flow, Europhys. Lett. 75, 750 (2006).

[30] S. Mittal, Global linear stability analysis of time-averaged flows, Int. J. Numer. Methods Fluids 58, 111 (2008).

[31] S. E. Turton, L. S. Tuckerman, and D. Barkley, Prediction of frequencies in thermosolutal convection from mean flows, Phys. Rev. E 91, 043009 (2015).

[32] V. Mantič-Lugo, C. Arratia, and F. Gallaire, Self-Consistent Mean Flow Description of the Nonlinear Saturation of the Vortex Shedding in the Cylinder Wake, Phys. Rev. Lett. 113, 084501 (2014).

[33] V. Mantič-Lugo, C. Arratia, and F. Gallaire, A self-consistent model for the saturation dynamics of the vortex shedding around the mean flow in the unstable cylinder wake, Phys. Fluids 27, 074103 (2015).

[34] F. Hecht, O. Pironneau, A. Le Hyaric, and K. Ohtsuka, FreeFem++ manual, 3rd ed., version 3.20, Tech. Rep. (Université Pierre et Marie Curie, Paris, 2014). 


\section{SATURATION OF THE RESPONSE TO STOCHASTIC ...}

[35] B. J. A. Zielinska, S. Goujon-Durand, J. Dusek, and J. E. Wesfreid, Strongly Nonlinear Effect in Unstable Wakes, Phys. Rev. Lett. 79, 3893 (1997).

[36] W. H. Press, S. A. Teukolsky, W. T. Vetterling, and B. P. Flannery, Numerical Recipes: The Art of Scientific Computing, 3rd ed. (Cambridge University Press, Cambridge, 2007), Vol. 1.

[37] N. Noiray, D. Durox, T. Schuller, and S. Candel, A unified framework for nonlinear combustion instability analysis based on the flame describing function, J. Fluid Mech. 615, 139 (2008).

[38] G. Berkooz, P. Holmes, and J. Lumley, The proper orthogonal decomposition in the analysis of turbulent flows, Annu. Rev. Fluid Mech. 25, 539 (1993).

[39] W. V. R. Malkus, Outline of a theory of turbulent shear flow, J. Fluid Mech. 1, 521 (1956).

[40] J. T. Stuart, On the non-linear mechanics of hydrodynamic stability, J. Fluid Mech. 4, 1 (1958). 